\title{
Adsorption characteristics of 1,2,4-trichlorobenzene, 2,4,6- trichlorophenol, 2-naphthol and naphthalene on graphene and graphene oxide
}

\author{
Zhiguo Pei ${ }^{a, *}$, Lingyun $\mathrm{Li}^{b}$, Lixiang Sun ${ }^{c}$, Shuzhen Zhang ${ }^{a}$, Xiao-quan Shan ${ }^{a}$, \\ Shuang Yang ${ }^{a}$, Bei Wen ${ }^{a}$ \\ a State Key Laboratory of Environmental Chemistry and Ecotoxicology, Research Center for Eco-Environmental Sciences, \\ Chinese Academy of Sciences, P.O. Box 2871, Beijing 100085, China \\ b Supervision and Testing Center for Vegetable Quality, Ministry of Agriculture, The Institute of Vegetables and Flowers, \\ Chinese Academy of Agricultural Sciences, Beijing, China \\ c College of Chemistry and Materials Science, Ludong University, Yantai 264025, China
}

\section{A R T I C L E I N F O}

Article history:

Received 9 May 2012

Accepted 10 August 2012

Available online 19 August 2012

\begin{abstract}
A B S T R A C T
Adsorption of 1,2,4-trichlorobenzene (TCB), 2,4,6-trichlorophenol (TCP), 2-naphthol and naphthalene (NAPH) on graphene $(G)$ and graphene oxide (GO) was investigated using a batch equilibration method and micro-Fourier transform infrared spectroscopy. All adsorption isotherms of four aromatics on G and GO were nonlinear, indicating that except for hydrophobic interaction, some specific interactions were involved in adsorption. For $G$, four aromatics had similar adsorption capacity at $\mathrm{pH} 5.0$ in despite of their different chemical properties. A series of $\mathrm{pH}$-dependent experimental results showed that 2-naphthol had higher adsorption capacity on $\mathrm{G}$ at alkaline $\mathrm{pH}$ than that at acidic $\mathrm{pH}$. Theoretical calculation ascribed this to higher $\pi$-electron density of anionic 2-naphthol than that of neutral 2naphthol, which facilitated the $\pi-\pi$ interaction formation with G. For GO, the adsorption affinity of four aromatics increased in the order: NAPH $<\mathrm{TCB}<\mathrm{TCP}<2$-naphthol. FTIR results revealed that TCB, TCP and 2-naphthol were adsorbed on G mainly via $\pi-\pi$ interaction. In contrast, high adsorption of TCP and 2-naphthol on GO was attributed to the formation of H-bonding between hydroxyl groups of TCP and 2-naphthol and O-containing functional groups on GO.
\end{abstract}

(c) 2012 Elsevier Ltd. All rights reserved.

\section{Introduction}

Industrialization and urbanization result in discharge of toxic chemicals in natural surface water bodies [1], including persistent aromatic pollutants, which are widely detected in the effluents of pharmaceutical, petrochemical, dyestuff, pesticide, and other industries. Numerous studies focused on the efficient removal of these toxic pollutants from aqueous solutions such as by photocatalysis [2], adsorption [3], and elec- trolysis [4]. Among these approaches, adsorption has been found to be superior to other techniques due to its low-cost, high efficiency and easy of operation.

Graphene $(G)$ is a two-dimensional structure consisting of $s p^{2}$ hybridized carbons with only one atomic thickness, and has attracted considerable attention since the report of Novoselov et al. [5]. Due to their large theoretical specific surface area, remarkable electronic properties and high ability of modification, potential environmental applications of $G$ as

\footnotetext{
* Corresponding author: Fax: +86 1062923563.

E-mail address: peizg@rcees.ac.cn (Z. Pei).

0008-6223/\$ - see front matter @ $\odot 2012$ Elsevier Ltd. All rights reserved.

http://dx.doi.org/10.1016/j.carbon.2012.08.024
} 
superior adsorbent have been recognized for removal of organic and inorganic contaminants from water and gases [614]. For example, it was found that sulfonated $G$ was one of the most effective adsorbents for naphthalene and 1-naphthol $[6,7]$. Wu et al. [8] revealed the high adsorption of acrylonitrile, p-toluenesulfonic acid, 1-naphthalenesulfonic acid and methyl blue on G nanosheets. Ramesha et al. [9] found that exfoliated graphene oxide (GO) and reduced GO are effective adsorbents for anionic and cationic dyes. In addition, $G$ nanosheets also have high adsorption affinity for heavy metals, where the amount of active surface sites on $\mathrm{G}$ is an important factor influencing the adsorption of heavy metal ions. Previous works on arsenic adsorption onto magnetite reduced GO composites and $\mathrm{G}$ sheets $[10,11]$, and on $\mathrm{Hg}(\mathrm{II})$ adsorption to polypyrrole-reduced GO composites showed that G composites have a strong adsorption affinity for metal ions [12,13].

It should be noted that $\mathrm{G}$ is a good model adsorbent for understanding phenomena occurring at carbon/solution interface due to its knowable structures. However, to our knowledge, most of previous adsorption studies about G nanosheets were limited to phenomenon description and/or to their potential application for water cleaning. Questions on the underlying adsorption mechanisms of organic contaminants on $G$ have not been addressed although several theoretical calculation or simulation studies assumed that charge transfer process and $\pi-\pi$ interaction were possibly responsible for the adsorption of aromatics to $G$ [15-17]. These simulation studies were only limited to $G$ with $<204$ carbon atoms and 40 hydrogen atoms to simplify the adsorption system and to make the calculation or simulation practical [16]. Current knowledge believes that real synthesized $\mathrm{G}$ is not ideal, which often has some defect sites and there is a small portion of O-containing functional groups on the outermost surface [18]. In addition, solvent effects on adsorption were not considered in the theoretical calculations. Therefore, detailed experimental studies, especially spectroscopic evidences for the relevant mechanisms of adsorption, are strongly needed to characterize the interactions between G and aromatics with different structures.

In order to better understand the adsorption mechanisms we choose $\mathrm{G}$ and $\mathrm{GO}$ with different $\pi$-electron density and Ocontaining functional groups as adsorbents and 1,2,4-trichlorobenzene (TCB), 2,4,6-trichlorophenol (TCP), 2-naphthol and naphthalene (NAPH) as common representatives of aromatics with different structures. NAPH and TCB are structurally similar to 2-naphthol and TCP, but contain no polar hydroxyl group. The aims of this study were (i) to elucidate the adsorption of aromatics with different structures on $\mathrm{G}$ and GO with different physicochemical properties; and (ii) to provide an insight to the relevant adsorption mechanisms using a batch equilibration method and micro-Fourier transform infrared spectroscopy ( $\mu$-FTIR).

\section{Experimental section}

\subsection{Chemicals}

TCP (97.4\%), TCB (98\%), 2-naphthol (98\%), and NAPH (98\%) were purchased from Aldrich Chemical Co. Inc. (Milwaukee,
WI), and used as-received. Their selected properties are listed in Table 1. $\mathrm{NaNO}_{3}, \mathrm{NaOH}$ and $\mathrm{HNO}_{3}$ were of guarantee reagent grade. Methanol was of HPLC grade.

\subsection{Materials}

G and GO were synthesized by a modified Hummers method [19]. Briefly, graphite powder (10 g) and sodium nitrate (5 g) were mixed with sulfuric acid $(230 \mathrm{~mL})$ in an ice bath, and potassium permanganate ( $30 \mathrm{~g})$ was slowly added, and the mixture was kept at $274 \mathrm{~K}$ for $2 \mathrm{~h}$ with continuous agitation. The reaction was kept at $308 \pm 2 \mathrm{~K}$ for $30 \mathrm{~min}$ with gas release, and then $460 \mathrm{~mL}$ of deionized water was gradually added, bringing about violent effervescence. The temperature of the water bath was increased to $371 \mathrm{~K}$ and the reaction was maintained at this temperature for $40 \mathrm{~min}$ in order to increase the oxidation degree of GO product. The resultant bright-yellow suspension was further treated with a $\mathrm{H}_{2} \mathrm{O}_{2}$ solution (30 $\mathrm{mL}, 30 \%$ ) followed by centrifugation and careful washing to clean out remnant salt. The wet GO was dewatered by vacuum drying at $323 \mathrm{~K}$. G was achieved by a low-temperature expansion under high vacuum. The above prepared GO was put into a quartz tube, which was connected to a high vacuum pump. The tube temperature was increased to $473 \mathrm{~K}$ at a rate of $50 \mathrm{~K} / \mathrm{min}$, and kept at this temperature for $5 \mathrm{~h}$ under a high vacuum $(<1 \mathrm{~Pa})$. After that the tube was cool down to room temperature and uninstalled from the vacuum pump, the produced $\mathrm{G}$ was obtained.

The surface functional groups on G and GO were determined by $\mu$-FTIR and X-ray photoelectron spectroscopy (XPS). Nitrogen specific surface areas of $G$ and GO were estimated using an accelerated surface area system and Brunauer-Emmett-Teller (BET) equation to adsorption of $\mathrm{N}_{2}$ at $77 \mathrm{~K}$.

\subsection{Adsorption experiments}

All adsorption experiments were conducted in triplicates using a batch equilibration technique at $23 \pm 1{ }^{\circ} \mathrm{C}$. Adsorption of TCB $(1.0,2.0,5.0,10.0,15.0,20.0$, and $27.0 \mathrm{mg} / \mathrm{L})$, TCP $(1.0,2.0$, 5.0, 10.0, 15.0, 20.0, and $27.0 \mathrm{mg} / \mathrm{L})$, 2-naphthol (1.0, 2.0, 5.0, 10.0, 15.0, 20.0, and $30.0 \mathrm{mg} / \mathrm{L})$, and NAPH $(2.0,5.0,10.0,15.0$, 20.0, and $25.0 \mathrm{mg} / \mathrm{L}$ ) were carried out by mixing a certain amount of $\mathrm{G}$ and $\mathrm{GO}$ with $0.01 \mathrm{M} \mathrm{NaNO}_{3}$ solution in $50 \mathrm{~mL}$ glass centrifuge tubes sealed with Teflon-lined screw caps. The suspended solution $\mathrm{pH}$ was adjusted to $5.0 \pm 0.1$ by addition of $0.1 \mathrm{M} \mathrm{HNO}_{3}$ or $0.1 \mathrm{M} \mathrm{NaOH}$ to assure these adsorbates present in neutral species during adsorption process. The suspension was stirred continuously for $24 \mathrm{~h}$ to ensure complete adsorption (apparent equilibrium was reached before this time). The suspension was centrifuged at $1667 \mathrm{~g}$ for $20 \mathrm{~min}$ to separate liquid from solid phases, and the concentrations of solutes in the supernatant phase were determined by HPLC. Controls were also prepared identically but contained no adsorbents, which were simultaneously run to assess loss of solutes. Results showed that no significant loss of solutes was observed (recoveries always $>99-101 \%$ ), indicating that microbial degradation, volatilization, or adsorption to the glass walls were negligible during the adsorption experiments.

The $\mathrm{pH}$ effect experiments were conducted using a batch adsorption approach as mentioned above. Both initial concen- 


\begin{tabular}{|c|c|c|c|c|c|}
\hline Organic chemicals & Structures & MW (g/mol) & $\mathrm{S}(\mathrm{mg} / \mathrm{L})$ & Log $K_{\text {ow }}$ & $\mathrm{pKa}$ \\
\hline $\mathrm{CP}$ & $\mathrm{OH}$ & 197.45 & 434.0 & 3.72 & 6.15 \\
\hline TCB & $\mathrm{Cl}$ & 181.5 & 31.0 & 4.25 & - \\
\hline 2-Naphthol & & 144.16 & 999.03 & 2.70 & 9.51 \\
\hline NAPH & & 128 & 32.13 & 3.33 & - \\
\hline
\end{tabular}

\footnotetext{
a MW: molecular weight, g/mol; $\mathrm{S}$ : aqueous solubility at room temperature, $\mathrm{mg} / \mathrm{L} ; \mathrm{K}_{\text {ow }}$ octanol-water partition coefficient; $\mathrm{pK}$, dissociation constant.
}

trations of four adsorbates were $5.0 \mathrm{mg} / \mathrm{L}$. After equilibrium was reached, the suspension was centrifuged, and the adsorbate concentrations in the supernatant were determined by HPLC. The adsorbed mass was calculated from the differences between the initial and final equilibrium concentrations.

\section{4. $\mu$-FTIR measurements and Raman spectra measurements}

$\mu$-FTIR spectra were recorded on NICOLET iN $10 \mathrm{MX}$ (Thermo Scientific). Samples for $\mu$-FTIR analysis were prepared with identical conditions to that used in the adsorption experiments. TCB-, TCP- or 2-naphthol-adsorbed G and GO were washed with $0.01 \mathrm{M} \mathrm{NaNO}_{3}$ solution, and then freeze-dried overnight. Microsamples were pressed on a diamond bracket and $\mu$-FTIR spectra were measured. The resolution for FTIR spectra was $16 \mathrm{~cm}^{-1}$, and a total of 100 scans were collected for each spectrum. Difference spectra of adsorbed samples are obtained by subtracting adsorbate free spectra of G or GO from the corresponding spectra of adsorbate adsorbed G or GO.

Raman spectra were recorded with a Renishew inVia Raman spectrometer (Renishaw plc, UK). The laser excitation was provided by an argon ion laser operated at wavelength of $514 \mathrm{~nm}$.

\subsection{Analysis}

Solutes in the supernatants were determined by an Agilent 1200 reversed-phase HPLC (Atlantis-dC18 ODS HPLC column, 4.6 i.d. $\times 150 \mathrm{~mm}, 5 \mu \mathrm{m}$ ) equipped with UV and fluorescence detectors. The determination wavelengths were set at $230 \mathrm{~nm}$ for TCB and TCP, the fluorescence wavelengths for measurements of 2-naphthol and NAPH were at excitation wavelengths of 275 and $225 \mathrm{~nm}$, and at emission wavelengths of 394 and $335 \mathrm{~nm}$, respectively. The mobile phase was methol:water (80:20, v:v) for TCB, TCP, and NAPH, and methol:water $\left(60: 40\right.$, v:v) for 2-naphthol at a flow rate of $1.0 \mathrm{~mL} \mathrm{~min}^{-1}$.

\subsection{Data analysis}

Adsorption data were fitted to the Freundlich equation using software Origin 7.5, and nonlinear regression analyses were conducted by the least-squares method. Freundlich equation can be expressed as $Q=K_{\mathrm{f}} \mathrm{C}^{\mathrm{n}}$, where $\mathrm{Q}(\mathrm{mg} / \mathrm{g})$ is the amount of adsorbed organic compounds by $\mathrm{G}$ and $\mathrm{GO}, \mathrm{C}(\mathrm{mg} / \mathrm{L})$ is the equilibrium concentration in solution, $K_{f}$ is an empirical constant related to adsorption capacity $\left(\mathrm{mg}^{(1-n)} \mathrm{g}^{-1} \mathrm{~L}^{\mathrm{n}}\right)$, and $n$ is the Freundlich exponent or a site energy heterogeneity factor, often used as an indicator of isotherm nonlinearity.

\section{Results and discussion}

\subsection{Characterization of $\mathrm{G}$ and GO}

Selected physical and chemical properties of G and GO are given in Table S1. The BET specific surface area of G and GO were found to be 239.1 and $65.0 \mathrm{~m}^{2} \mathrm{~g}^{-1}$, respectively. The discrepancy of the obtained surface area of $\mathrm{G}$ and theoretical value $\left(2620 \mathrm{~m}^{2} \mathrm{~g}^{-1}\right)$ is attributed to the incomplete exfoliation and aggregation during reduction process because of unavoidable van der Waals force between each single layer of G. Transmission electron microscopy (TEM) shows that both G and GO had typically wrinkled, sheet-like structures (Supplementary Fig. S1). 
In Fig. S2, Raman spectrum of $\mathrm{G}$ displays two prominent peaks at 1360 and $1593 \mathrm{~cm}^{-1}$, which correspond to the well documented $D$ band and $G$ band, respectively. It is well known that $G$ band corresponds to first-order scattering of $E_{2 g}$ mode observed for $\mathrm{sp}^{2}$ carbon domains, and pronounced $\mathrm{D}$ band is associated with structural defects, amorphous carbon, or edges that can break symmetry and selection rule. Defect content is given by the ratio of $\mathrm{D}$ band to $\mathrm{G}$ band intensity (ID/IG). Compared to GO, the ID/IG of G decreased from 0.79 to 0.68 , indicating decrease of amorphous carbon and structural defects on $\mathrm{G}$ layer (Fig. S2).

The FTIR spectra of G and GO are shown in Fig. S3. For G, peak at $1574 \mathrm{~cm}^{-1}$ was assigned to benzene ring $\mathrm{C}=\mathrm{C}$ stretching vibration. Peaks appeared at 1734 and $1240 \mathrm{~cm}^{-1}$ were the results of carboxyl $\mathrm{C}=\mathrm{O}$ and epoxy $\mathrm{C}-\mathrm{O}$ stretching vibrations, respectively. For GO, FTIR spectrum shows a broad peak at $3399 \mathrm{~cm}^{-1}$, which corresponds to $-\mathrm{OH}$ stretching vibration. In low wavenumbers range, FTIR spectra of GO shows $\mathrm{C}=\mathrm{O}$ $\left(1731 \mathrm{~cm}^{-1}\right)$, aromatic $\mathrm{C}=\mathrm{C}\left(1626 \mathrm{~cm}^{-1}\right)$, carboxyl $\mathrm{O}=\mathrm{C}-\mathrm{O}$ $\left(1378 \mathrm{~cm}^{-1}\right)$, and alkoxy C-O $\left(1073 \mathrm{~cm}^{-1}\right)$ stretching vibrations, respectively [10]. FTIR spectra indicated that not only $\mathrm{GO}$ but also $\mathrm{G}$ has some O-containing functional groups on their outermost surface and defect sites.

XPS provides useful information on the nature of functional groups and on the defects on the $G$ and GO surface. In Fig. 1a the XPS C1s peak of $\mathrm{G}$ is presented. Deconvolution of the C1s peak resolved a main peak of $\mathrm{sp}^{2} \mathrm{C}=\mathrm{C}(284.2 \mathrm{eV})$ and several small peaks of the carbon in C-O $(286.2 \mathrm{eV})$, the carbonyl carbon in $\mathrm{C}=\mathrm{O}(288.3 \mathrm{eV})$, and the carboxylate carbon in $\mathrm{O}-\mathrm{C}=\mathrm{O}(290.0 \mathrm{eV})$. The spectrum of GO showed four types of carbon bonds: the carbon $\mathrm{sp}^{2} \mathrm{C}=\mathrm{C}(284.8 \mathrm{eV})$ in the basal plane of GO, the carbon in $\mathrm{C}-\mathrm{O}(286.8 \mathrm{eV})$, the carbonyl carbon in $\mathrm{C}=\mathrm{O}(288.3 \mathrm{eV})$, and the carboxylate carbon in $\mathrm{O}-$ $\mathrm{C}=\mathrm{O}(290.2 \mathrm{eV}$ ) (Fig. 1b) $[8,20,21]$. These results indicated that the surface of GO had large numbers of functional groups. Compared to $\mathrm{G}$, the $\mathrm{C}-\mathrm{O}$ peak at $286.8 \mathrm{eV}$ and the $\mathrm{C}=\mathrm{O}$ peak at $288.3 \mathrm{eV}$ related to the oxidized carbon species were greatly strengthened, indicating that GO contained more O-containing functional groups than G.

\subsection{Effect of $\mathrm{pH}$ on adsorption}

Fig. 2 shows the effect of solution $\mathrm{pH}$ on the adsorption of TCB, TCP, 2-naphthol, and NAPH on G and GO. Generally, solu-
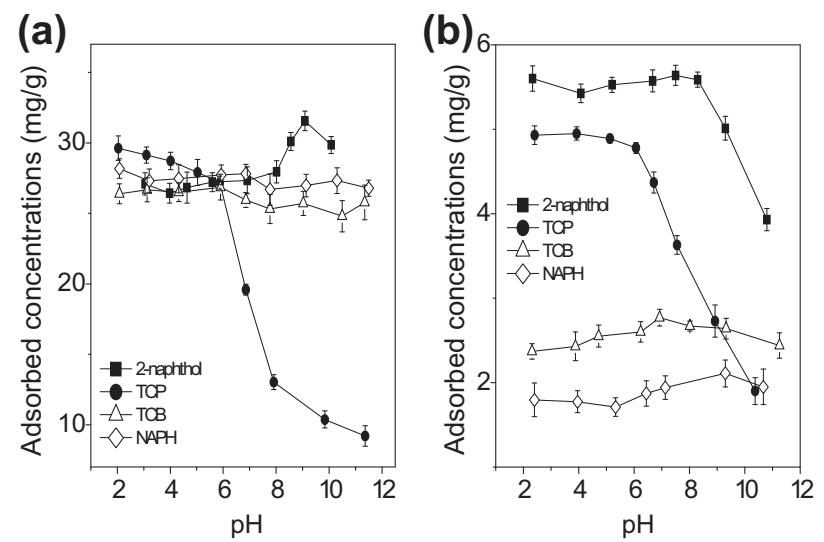

Fig. 2 - Effect of pH on the adsorption of TCP, TCB, 2naphthol, and NAPH on G (a) and GO (b).

tion $\mathrm{pH}$ had different effects on the adsorption of different solutes.

For TCB and NAPH, as shown in Fig. 2a and b, their adsorption on $\mathrm{G}$ and $\mathrm{GO}$ were almost independent of solution $\mathrm{pH}$ over the investigated $\mathrm{pH}$ range because both TCB and NAPH were nonionizable [3]. The adsorption of TCP decreased slightly over $\mathrm{pH}$ 2.0-6.0, and decreased rapidly at $\mathrm{pH}>6.0$, consistent to the adsorption of TCP on soil and peat [22]. Considering pKa value of TCP (6.15), it appears that the greater adsorption at low pH may be due to higher content of neutral TCP. An increase in solution $\mathrm{pH}$ increased the fraction of negatively charged TCP species. Therefore, electrostatic repulsion of anionic TCP with negatively charged surfaces of G and GO makes adsorption unfavorable.

A striking observation shown in Fig. 2 is that 2-naphthol exhibited remarkably different trends on $G$ and GO when solution $\mathrm{pH}$ changed. As shown in Fig. 2a, adsorption of 2naphthol on $\mathrm{G}$ was almost $\mathrm{pH}$-independent from $\mathrm{pH}$ 2.0-8.0, followed by a significant increase of adsorption capacity over $\mathrm{pH}$ range of 8.0-9.2, and then decreased slightly (Fig. 2a) [3,6]. In fact, even at $\mathrm{pH}$ 10.2, 2-naphthol still had considerably higher adsorption capacity on $\mathrm{G}$ than that at acidic pHs. Considering $\mathrm{pK}_{\mathrm{a}}$ value (9.51) of 2-naphthol, the predominant fraction of 2-naphthol was in anion form at $\mathrm{pH}$ above 9.51. The high adsorption capacity of 2-naphthol at higher $\mathrm{pH}$ suggested that specific adsorption-enhancement interaction
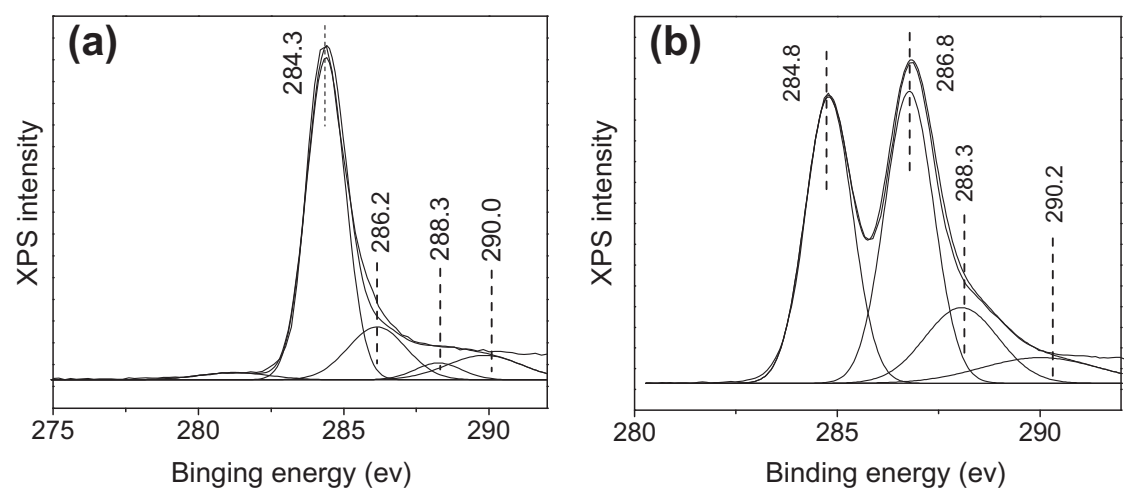

Fig. 1 - The C1s XPS spectra of G (a) and GO (b). 
occurred to counterbalance electrostatic repulsion between anionic 2-naphthol and G surface. Several possible mechanisms had been considered based on literatures: (1) hydrogen-bonding interaction between -OH group of 2-naphthol and O-containing groups on $G$; (2) direct anion- $\pi$ interaction between $-\mathrm{OH}$ or $-\mathrm{O}^{-}$(n-electron donor) groups of 2-naphthol and electron-depleted sites ( $\pi$-electron acceptor) of G [6]; (3) $\pi-\pi$ electron donor-acceptor (EDA) interaction between aromatic ring(s) of 2-naphthol and G surface [8]. Hydrogen-bonding was not likely a primary cause of the enhanced adsorption for following reason. At high $\mathrm{pH}$, both -OH group on 2-naphthol and O-containing groups of $\mathrm{G}$ would be ionized, and hydrogen-bonding effect should have been significantly impeded. However, Fig. 2a shows that adsorption affinity of 2 naphthol reached a maximum at $\mathrm{pH} \sim 9.2$. Zhao et al. [6] pointed out that ionized 1-naphthol might directly interact with the electron-depleted surface of sulfonated graphene via anion $-\pi$ interaction. But in this study, the anion $-\pi$ interaction was also excluded because adsorption of TCP was suppressed when its $-\mathrm{O}^{-}$group prevailed over its $-\mathrm{OH}$ group at high $\mathrm{pH}$. On the basis of above analysis, we propose that $\pi-$ $\pi$ EDA interaction between $\pi$-electron-rich naphthalene ring of 2-naphthol and $\pi$-electron-depleted regions of $\mathrm{G}$ surface was a primary mechanism for the enhanced adsorption of 2-naphthol, which would be verified in the following FTIR section. For 2-naphthol, its -OH group is a strong electron-donor, makes naphthalene ring of 2-naphthol $\pi$-electron rich, thus facilitating $\pi-\pi$ EDA interaction between 2-naphthol and G.
When -OH group of 2-naphthol dissociated to $-\mathrm{O}^{-}$at high $\mathrm{pH}$, it seemed that electron-donating strength would be further improved. The Mulliken electron distribution was calculated employing density functional theory [23-25] (B3LYP) with $6-311++G^{* *}$ basis set as implemented in the Gaussian 98 program [26]. Fig. 3 showed $\pi$-electron density of neutral and anionic 2-naphthol. It is found that the total Mulliken electrons on naphthalene ring of neutral 2-naphthol is about -1.038 e. When 2-naphthol is dissociated to anionic 2-naphthol, the total Mulliken electrons on naphthalene ring of 2naphthol is correspondingly increased to -1.208 e. Undoubtedly, an increase in $\pi$-electron density facilitated the adsorption of 2-naphthol to $\mathrm{G}$ at higher $\mathrm{pH}$ than that at lower $\mathrm{pH}$ despite electrostatic repulsion between anionic 2-naphthol and G surface. As for TCP, it has only one benzene ring and, accordingly, has much weaker $\pi$-electron-conjugating potential than two-ringed 2-naphthol. The total Mulliken electrons on benzene ring of neutral TCP are $-1.572 \mathrm{e}$ (Fig. 3). When TCP was dissociated to anion, the total Mulliken electrons on benzene ring did not increased as the same as 2-naphthol, while decreased $(-1.461 \mathrm{e})$. Possibly, this was one of reasons that TCP adsorption on $\mathrm{G}$ decreased at high $\mathrm{pH}$ range (Fig. 2a).

It should be noted that, if the same $\mathrm{pH}$ value is considered, four solutes has much lower adsorption capacity on GO than that on G (Fig. 2b), which is attributed to smaller surface area and less $\pi$-electron density of GO. Another possible reason is that hydrophilic GO surface containing various oxygen groups can bond water molecules, this competitive mechanism af-

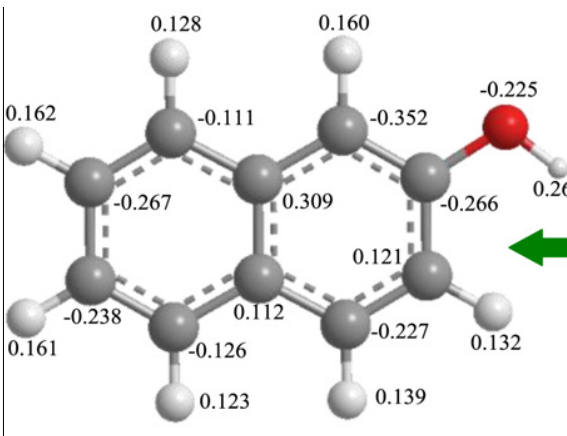

a

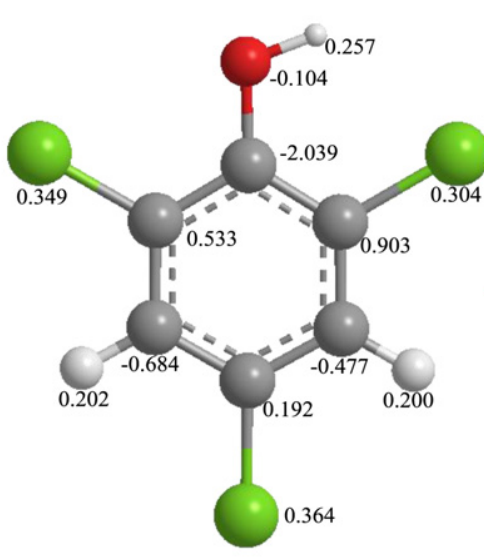

C

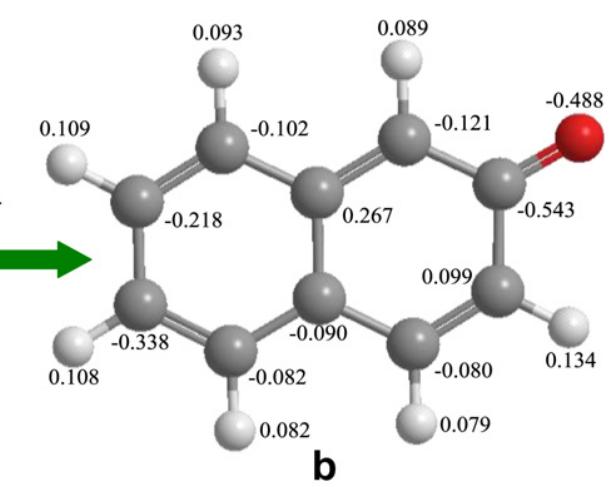

b

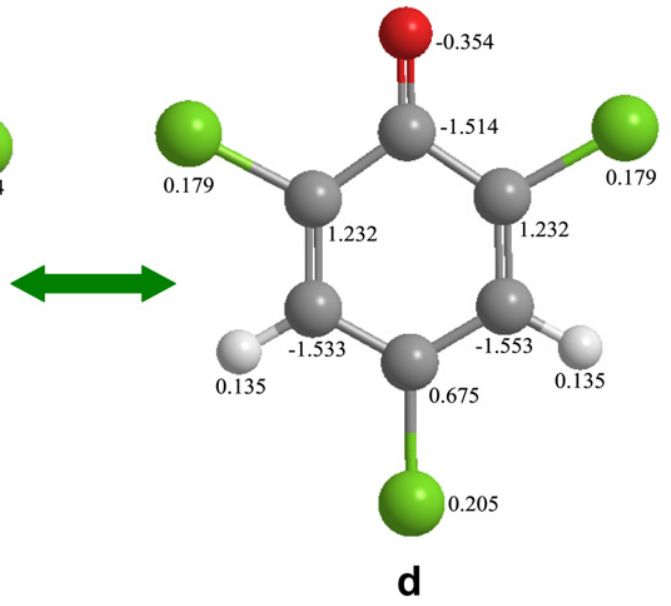

Fig. 3 - Mulliken electrons distribution schematics of neutral 2-naphthol (a), anionic 2-naphthol (b), neutral TCP (c), and anionic TCP (d). 

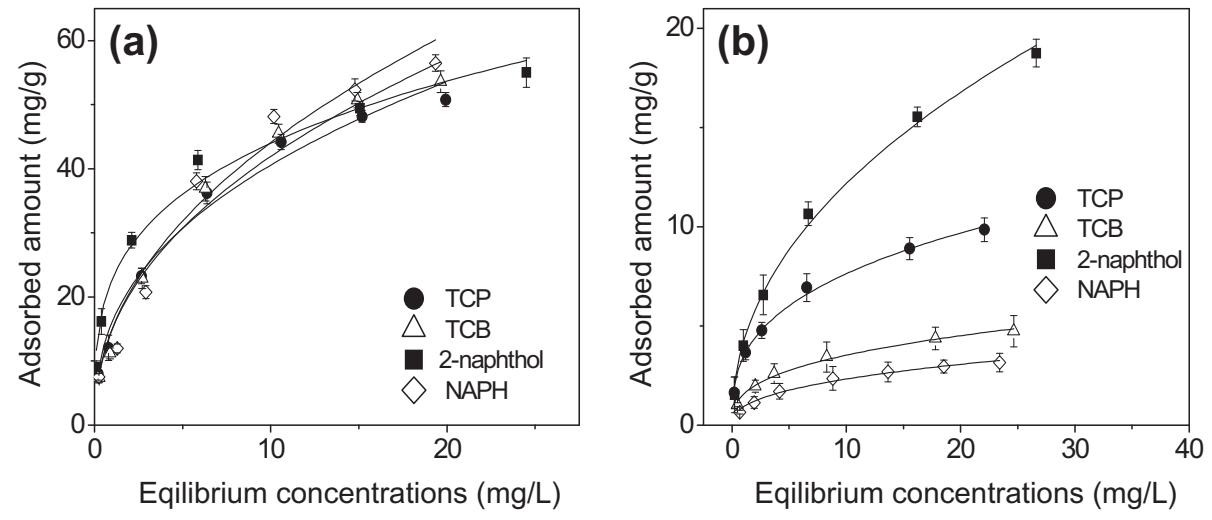

Fig. 4 - Adsorption isotherms of TCP, TCB, 2-naphthol, and NAPH on G (a) and GO (b).

fects the adsorption of other organic chemicals on GO. In contrast to G, GO has a markedly different effect on 2-naphthol adsorption as a function of solution $\mathrm{pH}$. Over $\mathrm{pH}$ 2.0-8.3, the adsorption of 2-naphthol on GO was $\mathrm{pH}$-independent. At $\mathrm{pH}>\sim 8.3$, the adsorption capacity of 2-naphthol was not increased as the same as that on $G$, while decreased rapidly. The different effects of $\mathrm{pH}$ on 2-naphthol adsorption are related to different properties of $\mathrm{G}$ from GO. Compared to $\mathrm{G}$, GO contained much less $\pi$-electron, and more O-containing groups on its surface, which was unfavorable for formation of $\pi-\pi$ interaction with 2-naphthol. Thus, 2-naphthol adsorption was not increased accordingly with increasing solution $\mathrm{pH}$ as the same as that on $\mathrm{G}$. This result also supported our above hypothesis that $\pi$-electron of $\mathrm{G}$ played an important role in the enhanced adsorption of 2-naphthol on G, especially at higher $\mathrm{pH}$ conditions.

\subsection{Adsorption isotherms}

The adsorption isotherms of TCB, TCP, 2-naphthanol, and NAPH on G and GO are shown in Fig. $4 a$ and b. All isotherms fitted Freundlich equation well. In general, all adsorption isotherms were highly nonlinear, and the regression parameters are listed in Table 2.

Fig. 4 a compares the adsorption of four adsorbates to G. It was found that, interestingly, adsorption behaviors of four adsorbates to $G$ were similar in despite of their different chemical properties. The adsorption was correlated poorly with hydrophobicity of these chemicals: water solubility $\left(C_{\text {sat }}\right)$ and $n$-octanol-water partition coefficient $\left(K_{\text {ow }}\right)$ (Table 1$)$. Similarly, the nonlinear adsorption isotherms of four adsorbates on $\mathrm{G}$ also suggested except for hydrophobic interaction, some specific interactions should be responsible for their adsorption on G. As described above, $\pi-\pi$ EDA interaction between aromatic ring of four adsorbates and $G$ surface should be a main mechanism responsible for strong adsorption of these adsorbates on G [6-8]. Compared to molecular structures of TCB and NAPH, TCP and 2-naphthol have a more hydroxyl group on their individual molecular structure, while their similar adsorption capacity on $\mathrm{G}$ indicated that the presence of hydroxyl group had no substantial contribution to the overall adsorption of TCP and 2-naphthol at the examined $\mathrm{pH}$. These results are consistent to Zhao et al., who also found NAPH and 1-naphthol had similar adsorption capacity on sulfonated graphene [6].

Different from $G$, adsorption of four adsorbates on GO followed the order of NAPH $<\mathrm{TCB}<\mathrm{TCP}<2$-naphthol, which indicated that different adsorption mechanisms should be responsible for the adsorption on GO (Fig. 4b). The strong adsorption of hydroxyl-substituted 2-naphthol and TCP indicated that their adsorption reaction was related to the presence of hydroxyl group. It was well known that GO has less $\pi$-electron and more O-containing groups on its surface. Thus, hydroxyl groups of 2-naphthol and TCP could interact with O-containing groups on GO through H-bonding. Following $\mu$-FTIR spectroscopic study provides a detailed insight to the adsorption mechanisms of these adsorbates on $G$ and GO.

Table 2 - Freundlich constants $\left(\mathrm{K}_{\mathrm{f}}\right.$ and $n$ ) and correlation coefficients $(r)$ of TCB, TCP, 2-naphthol, and NAPH adsorption onto G and GO $(n=3)$.

\begin{tabular}{|c|c|c|c|c|}
\hline Adsorbents & Adsorbates & $K_{\mathrm{f}}$ & $n$ & $r$ \\
\hline \multirow[t]{4}{*}{ G } & TCP & $16.10(1.65)$ & $0.40(0.04)$ & 0.97 \\
\hline & TCB & $15.01(1.60)$ & $0.40(0.04)$ & 0.97 \\
\hline & 2-Naphthol & $22.70(1.65)$ & $0.29(0.03)$ & 0.99 \\
\hline & NAPH & $14.17(1.55)$ & $0.47(0.04)$ & 0.97 \\
\hline \multirow[t]{4}{*}{ GO } & TCP & $3.46(0.16)$ & $0.34(0.02)$ & 0.99 \\
\hline & TCB & $1.56(0.16)$ & $0.35(0.04)$ & 0.98 \\
\hline & 2-Naphthol & $4.20(0.15)$ & $0.53(0.01)$ & 0.99 \\
\hline & NAPH & $0.93(0.16)$ & $0.40(0.06)$ & 0.99 \\
\hline
\end{tabular}



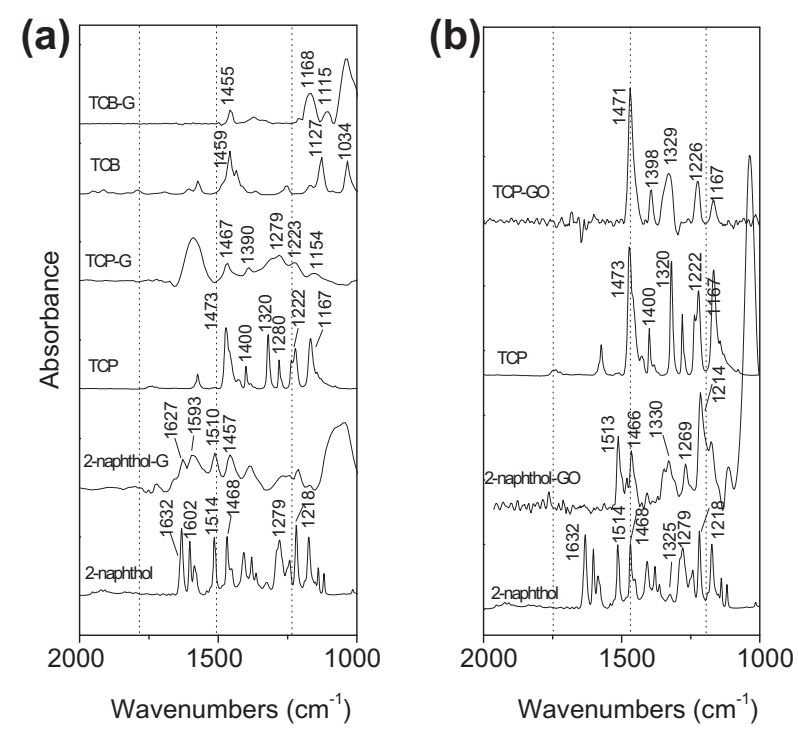

Fig. 5 - $\mu$-FTIR spectra of pure TCP, TCB, and 2-naphthol, and adsorbed TCP, TCB, and 2-naphthol on G (a) and GO (b), respectively.

\section{4. $\mu$-FTIR analysis}

To reveal the adsorption mechanisms of four adsorbates on $G$ and GO, a $\mu$-FTIR study was performed. The $\mu$-FTIR spectra of TCB-G, TCB, TCP-G, TCP, 2-naphthol-G, and 2-naphthol are shown in Fig. 5a. For TCB, the prominent peaks at $1459 \mathrm{~cm}^{-1}$ are attributed to benzene ring $\mathrm{C}=\mathrm{C}$ stretching, and the band at $1127 \mathrm{~cm}^{-1}$ is due to strong C-C skeletal vibrations of benzene ring [27]. For TCP, peaks at 1473, 1400, and $1167 \mathrm{~cm}^{-1}$ are assigned to $\mathrm{C}=\mathrm{C}$ stretching vibration and $\mathrm{C}-\mathrm{C}$ skeletal vibrations of benzene ring. Peak at $1280 \mathrm{~cm}^{-1}$ is assigned to C-O stretching vibration. Two bands appeared at 1320 and $1222 \mathrm{~cm}^{-1}$ are attributed to $\mathrm{O}-\mathrm{H}$ bending and deformation vibrations [28]. After TCB and TCP were adsorbed on G, peaks of $\mathrm{C}=\mathrm{C}$ stretching vibration and $\mathrm{C}-\mathrm{C}$ skeletal vibrations of benzene ring shifted from 1459 and $1127 \mathrm{~cm}^{-1}$ to 1455 and $1115 \mathrm{~cm}^{-1}$ for TCB, and shifted from 1473, 1400, and $1167 \mathrm{~cm}^{-1}$ to 1467,1390 , and $1154 \mathrm{~cm}^{-1}$ for TCP, respectively. Such shifts suggested that benzene rings of TCB and TCP attended the whole adsorption process on $G$ through $\pi-\pi$ interaction. It was significant that $\pi-\pi$ interaction weakened the double band character of benzene ring and caused red-shift of $\mathrm{C}=\mathrm{C}$ stretching vibration bands (Fig. $5 \mathrm{a}$ ). Previous theoretical calculation suggested that benzoic acid adsorption on $G$ was driven primarily by electron transfer process between the $\mathrm{G}$ surface and the $-\mathrm{COOH}$ group, and to lesser extent by $\pi-\pi$ interaction between the $\mathrm{G}$ surface and the aromatic core of benzoic acid [16]. However, in this study, for TCP with electron-donating-OH group, different observation was obtained. As shown in Fig. $5 \mathrm{a}$, the $\mathrm{C}-\mathrm{O}$ stretching vibration $\left(1280 \mathrm{~cm}^{-1}\right)$ and $\mathrm{O}-\mathrm{H}$ deformation vibration $\left(1320\right.$ and $1222 \mathrm{~cm}^{-1}$ ) of TCP have not been significantly shifted when TCP was adsorbed on $\mathrm{G}$, indicating that hydroxyl group was not involved in the adsorption reaction. It is also the reason why TCB and TCP have similar adsorption capacity on $G$ though they have different molecule structures. As for 2-naphthol standard, peaks at $1632,1602,1514$, and $1468 \mathrm{~cm}^{-1}$ are attributed to benzene ring $\mathrm{C}=\mathrm{C}$ stretching, and band at $1279 \mathrm{~cm}^{-1}$ is due to $\mathrm{C}-\mathrm{O}$ stretching vibration, respectively $[29,30]$. Two peaks at 1325 and $1218 \mathrm{~cm}^{-1}$ are attributed to $\mathrm{O}-\mathrm{H}$ bending and deformation vibrations of 2-naphthol [28]. When 2-naphthol was adsorbed on $\mathrm{G}$ (Fig. 5a), it was found that its $\mathrm{C}=\mathrm{C}$ stretching vibration peaks at 1632,1602 , and $1468 \mathrm{~cm}^{-1}$ were shifted to 1627,1593 , and $1457 \mathrm{~cm}^{-1}$, respectively, suggesting the occurrence of $\pi-\pi$ interaction in the form of face-to-face stacking between 2-naphthol and G surface. As for NAPH, no FTIR spectra are provided in Fig. $5 a$ due to its weak FTIR response signal in $1000-2000 \mathrm{~cm}^{-1}$. However, according to previous theoretical calculation [6], the main adsorption mechanism of NAPH on G was $\pi-\pi$ interaction, where NAPH was adsorbed in a tiled form.

Compared to G, FTIR results revealed that TCP and 2-naphthol have significantly different adsorption mechanism on GO (Fig. 5b). When TCP was adsorbed on GO, it was found that the $\mathrm{C}-\mathrm{C}$ skeletal vibrations $\left(1167 \mathrm{~cm}^{-1}\right)$ of benzene ring have no shift. And peaks of $\mathrm{C}=\mathrm{C}$ stretching vibration of benzene ring had very slight shifts from 1473 and $1400 \mathrm{~cm}^{-1}$ to 1471 and $1398 \mathrm{~cm}^{-1}$, respectively. Considering the resolution of $\mu$-FTIR, such slight shifts could not be used as evidence for $\pi-\pi$ interaction. In contrast, the $\mathrm{O}-\mathrm{H}$ bending vibration of TCP shifted significantly from 1320 to $1329 \mathrm{~cm}^{-1}$ when TCP was adsorbed on GO. This suggested that TCP was adsorbed on GO mainly through H-bonding between hydroxyl group of TCP and Ocontaining group on GO surface rather than $\pi-\pi$ interaction. Similarly, when 2-naphthol was adsorbed on GO, peaks attributable to $\mathrm{C}=\mathrm{C}$ stretching vibration (1514 and $1468 \mathrm{~cm}^{-1}$ ) exhibited no shift, suggesting that naphthalene ring of 2naphthol was not involved in adsorption process on GO (Fig. 5b). However, when C-O stretching vibration of adsorbed 2-naphthol at $1269 \mathrm{~cm}^{-1}$ was compared with that of 2-naphthol standard at $1279 \mathrm{~cm}^{-1}$, it appears that a $10 \mathrm{~cm}^{-1}$ shift resulted from the formation of $\mathrm{H}$-bonding between hydroxyl group of 2-naphthol and O-containing groups on GO. The shift of $\mathrm{O}-\mathrm{H}$ bending vibrations from 1325 to $1330 \mathrm{~cm}^{-1}$ also verified the presence of H-bonding. This is also the possible reason why TCP and 2-naphthol have higher adsorption capacities on GO than that of TCB and NAPH.

We also tried to give some FTIR evidences for the mechanisms of TCB and NAPH adsorption onto GO. Unfortunately, the obtained FTIR spectra were characterized by broad and overlapping bands that do not provide detailed clues as to identification of adsorption mechanisms due to their low adsorption capacities and/or FTIR response signal. Thus, no FTIR spectra of TCB and NAPH adsorption on GO are shown in Fig. $5 b$.

\section{Conclusions}

In view of the foregoing study, it was found that G and GO with different properties have different effects on four aromatics adsorption. On G, four aromatics had similar adsorption capacity at pH 5.0 in despite of their different chemical properties. It was indicated that except for hydrophobic interaction, four aromatics were adsorbed on G mainly via $\pi-\pi$ interaction. Compared to G, GO has lesser $\pi$-electron density 
and more O-containing functional groups. The adsorption affinity of four aromatics increased in the order $\mathrm{NAPH}<\mathrm{TCB}<\mathrm{TCP}<2$-naphthol on GO. The higher adsorption of hydroxyl-substituted TCP and 2-naphthol on GO was mainly attributed to the formation of $\mathrm{H}$-bonding between hydroxyl groups of TCP and 2-naphthol and O-containing functional groups on GO.

\section{Acknowledgment}

This work was funded by National Basic Research Program of China (Project 2011CB936001) and National Natural Science Foundation of China (Grant numbers: 41071308, 21277159, 40730740 and 21103080). Prof. Weng Shifu, College of Chemistry and Molecular Engineering, Peking University, was highly appreciated for his constructive discussion on FTIR data.

\section{Appendix A. Supplementary data}

Supplementary data associated with this article can be found, in the online version, at http://dx.doi.org/10.1016/j.carbon. 2012.08.024.

\section{R E F E R E N C E S}

[1] Li P, Wang X, Allinsonb G, Li X, Xiong X. Risk assessment of heavy metals in soil previously irrigated with industrial wastewater in Shenyang, China. J Hazard Mater 2009;161(1):516-21.

[2] Ide Y, Nakasato Y, Ogawa M. Molecular recognitive photocatalysis driven by the selective adsorption on layered titanates. J Am Chem Soc 2010;132(10):3601-4.

[3] Chen W, Duan L, Wang L, Zhu DQ. Adsorption of hydroxyland amino-substituted aromatics to carbon nanotubes. Environ Sci Technol 2008;42(18):6862-8.

[4] Zuman P, Ludvík J. The use of controlled potential electrolysis with a dropping mercury electrode in elucidation of organic electroreduction mechanisms. Electroanalysis 2000;12(12):879-88.

[5] Novoselov KS, Geim AK, Morozov SV, Jiang D, Zhang Y, Dubonos SV, et al. Electric field effect in atomically thin carbon films. Science 2004;306(5696):666-9.

[6] Zhao GX, Jiang L, He YD, Li JX, Dong HL, Wang XK, et al. Sulfonated graphene for persistent aromatic pollutant management. Adv Mater 2011;23(34):3959-63.

[7] Zhao GX, Li JX, Wang XK. Kinetic and thermodynamic study of 1-naphthol adsorption from aqueous solution to sulfonated graphene nanosheets. Chem Eng J 2011;173(1):185-90.

[8] Wu T, Cai X, Tan SZ, Li HY, Liu JS, Yang WD. Adsorption characteristics of acrylonitrile, p-toluenesulfonic acid, 1naphthalenesulfonic acid and methyl blue on graphene in aqueous solutions. Chem Eng J 2011;173(1):144-9.

[9] Ramesha GK, Vijaya Kumara A, Muralidhara HB, Sampath S. Graphene and graphene oxide as effective adsorbents toward anionic and cationic dyes. J Colloid Interface Sci 2011;361(1):270-7.
[10] Chandra V, Park J, Chun Y, Lee JW, Hwang IC, Kim KS. Waterdispersible magnetite-reduced graphene oxide composites for arsenic removal. ACS Nano 2010;4(7):3979-86.

[11] Mishra AK, Ramaprabhu MS. Functionalized graphene sheets for arsenic removal and desalination of sea water. Desalination 2011;282:39-45.

[12] Chandra C, Kim KS. Highly selective adsorption of $\mathrm{Hg}^{2+}$ by a polypyrrole-reduced graphene oxide composite. Chem Commun 2011;47(13):3942-4.

[13] Ren YM, Yan N, Wen Q Fan ZJ, Wei T, Zhang ML, et al. Graphene/ $\delta-\mathrm{MnO}_{2}$ composite as adsorbent for the removal of nickel ions from wastewater. Chem Eng J 2011;175:1-7.

[14] Cabrera-Sanfelix P. Adsorption and reactivity of $\mathrm{CO}_{2}$ on defective graphene sheets. J Phys Chem A 2009;113(2):493-8.

[15] AlZahrani AZ. First-principles study on the structural and electronic properties of graphene upon benzene and naphthalene adsorption. Appl Surf Sci 2010;257(3):807-10.

[16] Rochefort A, Wuest JD. Interaction of substituted aromatic compounds with graphene. Langmuir 2009;25(1):210-5.

[17] Kozlov SM, Vines F, Görling A. On the interaction of polycyclic aromatic compounds with graphene. Carbon 2012;50(7):2482-92.

[18] Lin DH, Xing BS. Adsorption of phenolic compounds by carbon nanotubes: role of aromaticity and substitution of hydroxyl groups. Environ Sci Technol 2008;42(19):7254-9.

[19] Lv W, Tang DM, He YB, You CH, Shi ZQ, Chen XC, et al. Lowtemperature exfoliated graphenes: vacuum-promoted exfoliation and electrochemical energy storage. ACS Nano 2009;3(11):3730-6.

[20] Park S, An JH, Jung IW, Piner RD, An SJ, Li XS, et al. Colloidal suspensions of highly reduced graphene oxide in a wide variety of organic solvents. Nano Lett 2009;9(4):1593-7.

[21] Datsyuk V, Kalyva M, Papagelis K, Parthenios J, Tasis D, Siokou A, et al. Chemical oxidation of multi walled carbon nanotubes. Carbon 2008;46(6):833-40.

[22] Pei ZG, Shan XQ Liu T, Xie YN, Wen B, Zhang SZ, et al. Effect of lead on the sorption of 2,4,6-trichlorophenol on soil and peat. Environ Pollut 2007;147(3):764-70.

[23] Becke AD. Density-functional thermochemistry. III. the role of exact exchange. J Chem Phys 1993;98(7):5648-52.

[24] Lee C, Yang W, Parr RG. Development of the colle-salvetti correlation-energy formula into a functional of the electron density. Phys Rev B 1988;37(2):785-9.

[25] Stephens PJ, Devlin FJ, Chabalowski CF, Frisch MJ. Ab initio calculation of vibrational absorption and circular dichroism spectra using density functional force fields. J Phys Chem 1994;98(45):11623-7.

[26] Frisch MJ, Trucks GW, Schlegel HB, Scuseria GE, Robb MA, Cheeseman JR, et al. Gaussian 98. Pittsburgh, PA: Gaussian, Inc.; 1998.

[27] Udayakumar V, Periandy S, Ramalingam S. Experimental (FTIR and FT-Raman) and theoretical (HF and DFT) investigation, IR intensity, Raman activity and frequency estimation analyses on 1-bromo-4-chlorobenzene. Spectrochimica Acta Part A 2011;79(5):920-7.

[28] Kung KHS, McBride MB. Bonding of chlorophenols on iron and aluminum oxides. Environ Sci Technol 1991;25(4):702-9.

[29] Karthikeyan KG, Chorover J, Bortiatynski JM, Hatcher PG. Interaction of 1-naphthol and its oxidation products with aluminum hydroxide. Environ Sci Technol 1999;33(22):4009-15.

[30] Janarthanan S, Sugaraj Samuel R, Selvakumar S, Rajan YC, Jayaraman D, Pandi S. Growth and characterization of organic NLO crystal $\beta$-naphthol. J Mater Sci Technol 2011;27(3):271-4. 